\title{
Electrodeposition of GeSbTe Based Resistive Switching Memory in Crossbar Arrays
}

Ayoub H. Jaafar, ${ }^{a, *}$ Lingcong Meng, ${ }^{b, c}$ Yasir J. Noori, ${ }^{a}$ Wenjian Zhang, ${ }^{b}$ Yisong Han, ${ }^{d}$ Richard

Beanland, ${ }^{d}$ David C. Smith, ${ }^{e}$ Gillian Reid,${ }^{b}$ Kees de Groot ${ }^{a}$ Ruomeng Huang, ${ }^{a,}{ }^{*}$ and Philip N.

Bartlett $^{b, *}$

${ }^{a}$ School of Electronics and Computer Science, University of Southampton, Southampton, SO17 1BJ, UK

${ }^{b}$ School of Chemistry, University of Southampton, Southampton, SO17 1BJ, UK

${ }^{c}$ School of Chemistry, University of Lincoln, Lincoln, LN6 7TS, UK

${ }^{d}$ Department of Physics, University of Warwick, Coventry, CV4 7AL, UK

${ }^{e}$ School of Physics, University of Southampton, Southampton, SO17 1BJ, UK

*a.h.j.hamdiyah@soton.ac.uk; r.huang@soton.ac.uk; p.n.bartlett@soton.ac.uk

\section{Supplementary Figures}




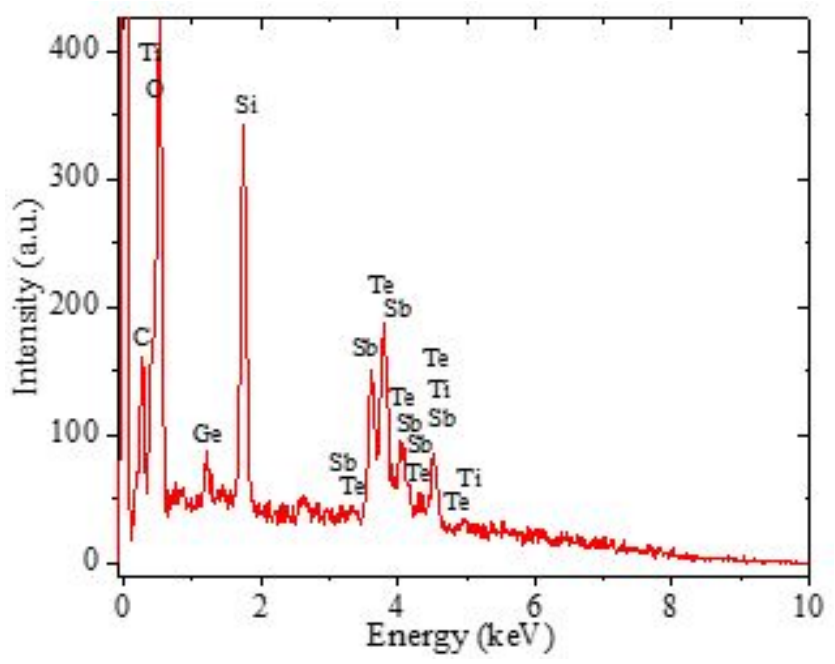

Figure S1. EDX spectrum of the electrodeposited GeSbTe showing the existence of Ge in the GeSbTe crossbar array. The EDX spectrum shows the presence of $\mathrm{Cl}$ inside the cell, which is originated from the electrolyte.
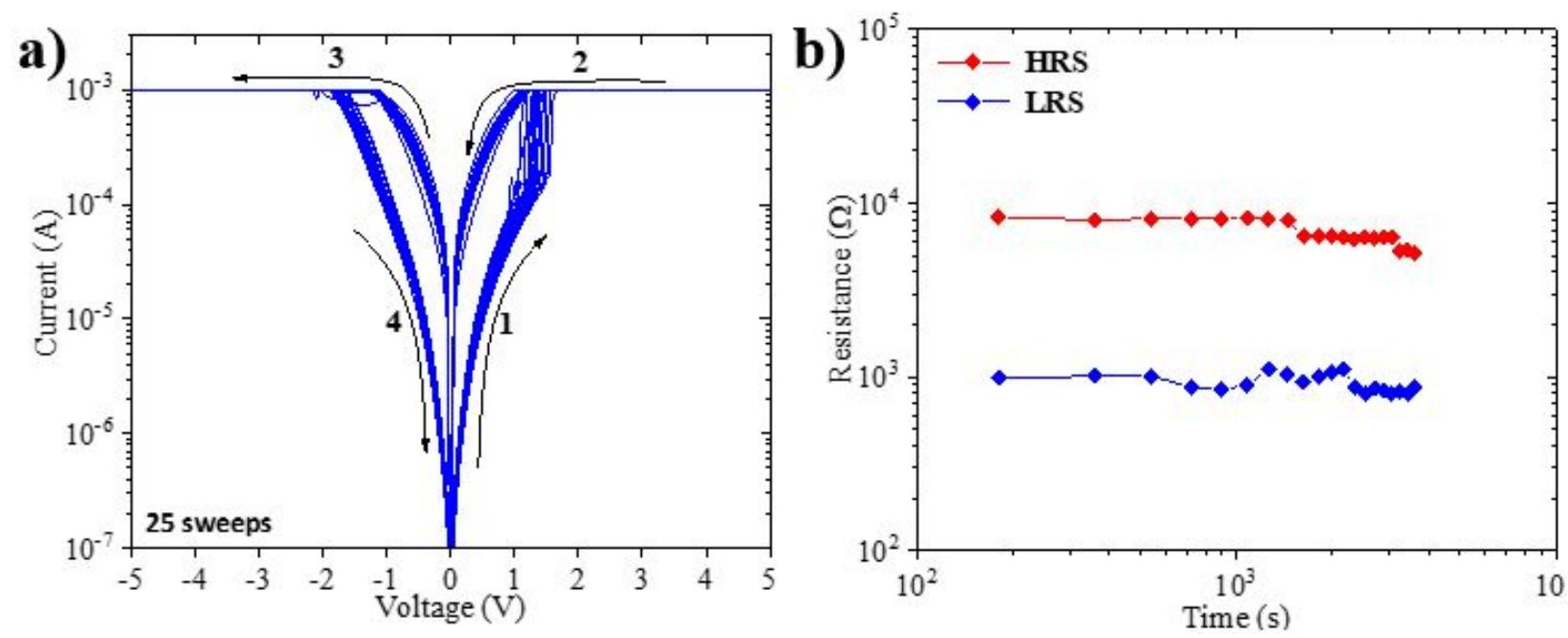

Figure S2. (a) Consecutive I-V characteristics of an electrodeposited crossbar TiN/GeSbTe/TiN memory cell swept between $-5 \mathrm{~V}$ and $5 \mathrm{~V}$ with $\mathrm{CC}$ of $1 \mathrm{~mA}$. The graph clearly shows a uniform and reproducible resistive switching properties of the memory cell. (b) Retention data test for a 
TiN/GeSbTe/TiN memory cell in both the HRS and LRS measured at $0.2 \mathrm{~V}$ for 1 hour without considerable resistance degradation.
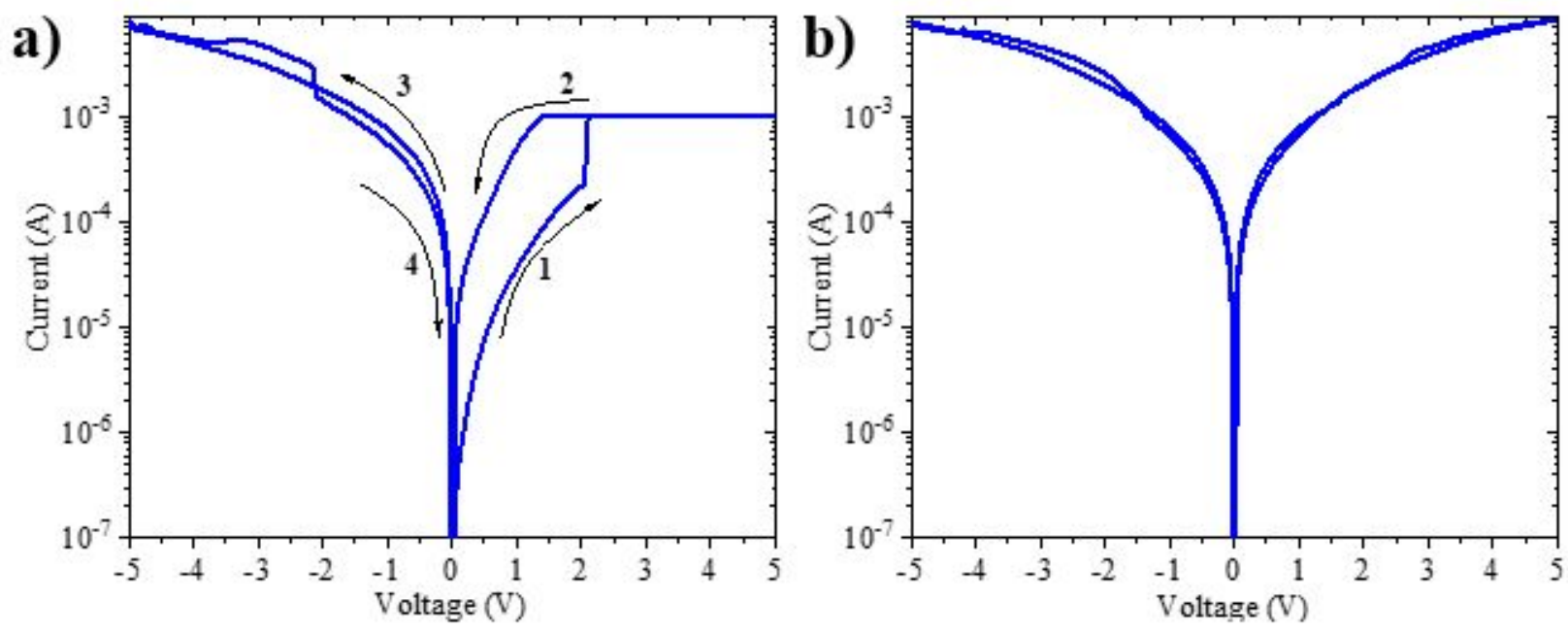

Figure S3. I-V characteristics of an electrodeposited crossbar TiN/GeSbTe/TiN memory, showing the impact of absence of the CC during the RESET process on the resistive switching properties.

(a) I-V curve shows that the cell switches to a higher operating current value, in which the cell cannot be switched back to the HRS by application of a negative sweep voltage. (b) The subsequent I-V curve shows the diminishing of the resistive switching properties of the cell. 


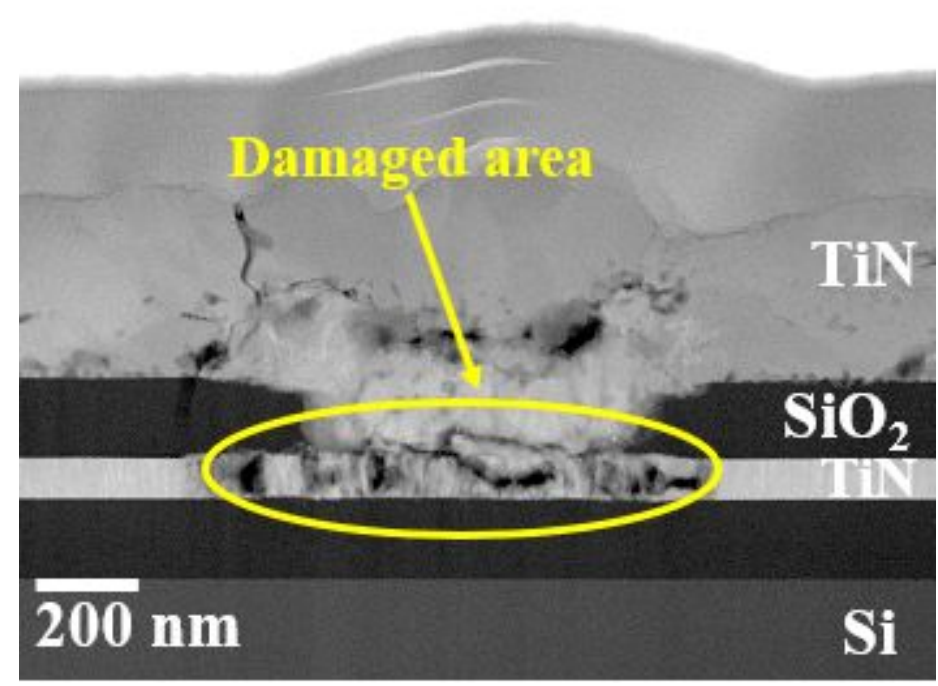

Figure S4. TEM image of a damaged device after breakdown. The large Joule heat generated in the absence of the CC during the RESET process caused permanent damage to both the GeSbTe layer and the TiN electrodes.

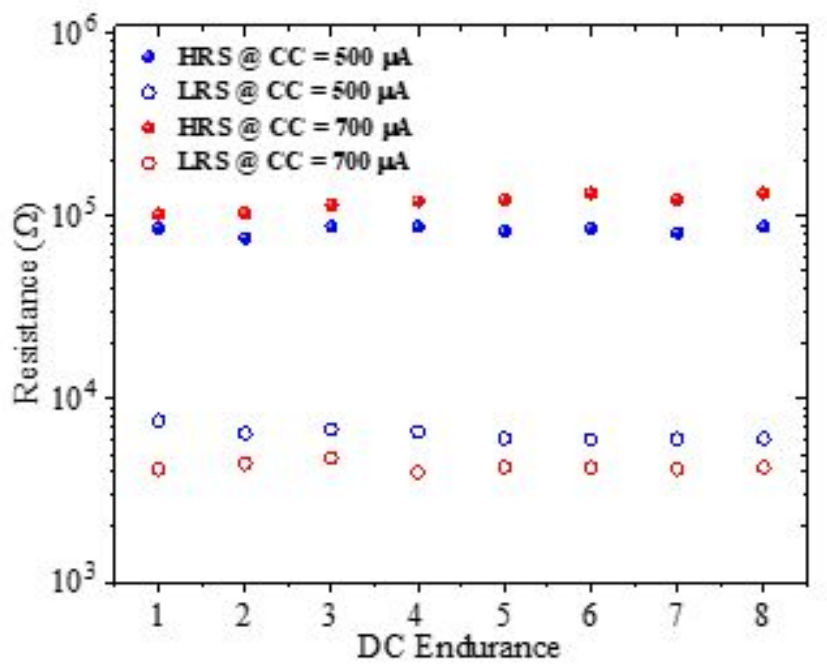

Figure S5. Multi-state resistance behavior of a TiN/GeSbTe/TiN memory cell by varying the CC from $500 \mu \mathrm{A}$ (blue data) to $700 \mu \mathrm{A}$ (red data). It can be observed that changing the $\mathrm{CC}$ resulted in 
the variation of LRS and HRS. ${ }^{1,2}$ The resistance values of both LRS and HRS were measured at $0.5 \mathrm{~V}$.

\section{References}

(1) Nagashima, K.; Yanagida, T.; Oka, K.; Taniguchi, M.; Kawai, T.; Kim, J. S.; Park, B. H. Resistive Switching Multistate Nonvolatile Memory Effects in a Single Cobalt Oxide Nanowire. Nano Lett. 2010, $10(4), 1359-1363$.

(2) Niu, G.; Schubert, M. A.; Sharath, S. U.; Zaumseil, P.; Vogel, S.; Wenger, C.; Hildebrandt, E.; Bhupathi, S.; Perez, E.; Alff, L.; Lehmann, M.; Schroeder, T.; Niermann, T. Electron Holography on HfO2/HfO2-x Bilayer Structures with Multilevel Resistive Switching Properties. Nanotechnology 2017, 28 (21). 